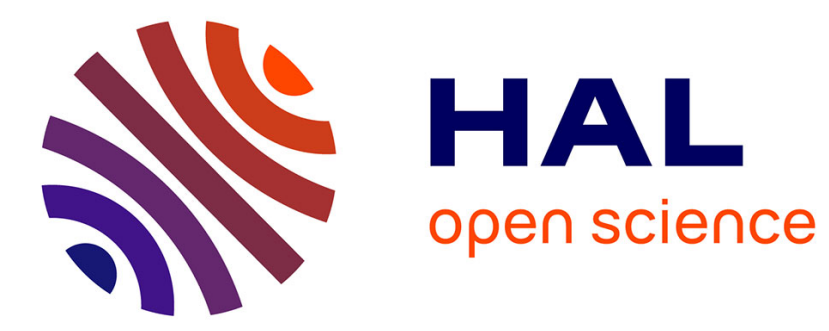

\title{
Perversion narcissique, genre et conjugalité Pauline Delage
}

\section{To cite this version:}

Pauline Delage. Perversion narcissique, genre et conjugalité. Zilsel: science, technique, société, 2021, 8, pp.240-253. 10.3917/zil.008.0240 . hal-03480058

\section{HAL Id: hal-03480058 https://hal.science/hal-03480058}

Submitted on 3 Jan 2022

HAL is a multi-disciplinary open access archive for the deposit and dissemination of scientific research documents, whether they are published or not. The documents may come from teaching and research institutions in France or abroad, or from public or private research centers.
L'archive ouverte pluridisciplinaire HAL, est destinée au dépôt et à la diffusion de documents scientifiques de niveau recherche, publiés ou non, émanant des établissements d'enseignement et de recherche français ou étrangers, des laboratoires publics ou privés. 


\title{
Perversion narcissique, genre et conjugalité
}

\author{
Pauline Delage ${ }^{1}$
}

$\mathrm{Au}$ détour d'une note de bas de page de l'article "La femme du pervers narcissique », la psychanalyste Simone Korff-Sausse se dit «frappée par le fait que les descriptions des praticiens qui s'occupent de situations de violences conjugales, et en particulier ceux qui ont mis en place des dispositifs d'accueil pour femmes battues (Bin-Heng, Cherbit et Lombardi, 1996 ; Gillioz, De Puy et Ducret, 1997) correspondent jusqu'au moindre détail à [s]es propres observations ${ }^{2} »$. Traiter la violence conjugale, de Mary Bin-Heng, Framboise Cherbit et Édith Lombardi, et Domination et violence envers la femme dans le couple, de Lucienne Gillioz, Jacqueline de Puy et Véronique Ducret, les références citées, renvoient à deux des principaux ouvrages francophones publiés dans les années 1990 par des actrices féministes de la lutte contre la violence conjugale. Pour autant, rien n'est dit des rapports de domination et de pouvoir qui se jouent dans le couple hétérosexuel.

En France, l'expression « perversion narcissique » est souvent utilisée comme synonyme de violences psychologiques : quoique renvoyant à d'autres sphères que le couple, dont le travail, la catégorie désigne souvent le cadre conjugal. "Pervers narcissique » circule également largement, dans les médias notamment, pour désigner ceux qui seraient plus volontiers qualifiés, dans les politiques publiques et les associations d'aide aux victimes, d'" auteurs de violences psychologiques » dans le couple. À titre d'exemple, le court-métrage « Fred et Marie », lancé par la Direction pour l'Égalité des Chances en 2011 dans le cadre d'une campagne de sensibilisation contre les violences psychologiques en Belgique francophone, et utilisé par certaines organisations en France, comme le Centre Hubertine Auclert, a été présenté par le journal L'Express comme étant « le spot qui dénonce les pervers narcissiques $»^{3}$.

Aussi la perversion narcissique et les pervers narcissiques sont-ils « à la mode ${ }^{4}$ : ces deux notions sont suffisamment élastiques pour être à la fois utilisées par des spécialistes, formé·e·s à la psychologie et à la psychanalyse, et intégrées au sens commun, comme catégorie profane qui circule notamment médiatiquement. Être un pervers narcissique, c'est utiliser sa posture et son pouvoir pour rabaisser autrui, s'en nourrir, et finalement le détruire psychiquement. Ainsi, la notion peut parler à tout le monde ; et elle peut parler de tout le monde, hommes ou femmes.

Si certaines catégories, comme celles de violences sexistes, de violences envers les femmes, voire, par son histoire, de violences conjugales, permettent de refléter l'asymétrie de genre au cœur des violences interpersonnelles dans le couple, la perversion narcissique, en soi, laisse largement dans le flou l'influence du genre sur les relations intimes. Cependant, en recoupant en partie le sens des catégories élaborées par des mouvements associatifs pour dénoncer les violences sexistes ${ }^{5}$, la perversion narcissique s'inscrit dans un ensemble de discours sur les rapports de genre dans le couple hétérosexuel, et contribue à les souligner, les nuancer, ou les dénier.

Cet article cherche à dresser quelques pistes pour saisir ce que l'usage de «pervers narcissique » révèle de la compréhension des rapports de genre ainsi que des normes conjugales. Pour cela, nous reviendrons tout d'abord sur les travaux pionniers de Marie-France Hirigoyen dont le manque de systématicité dans l'appréhension du genre laisse parfois place à une conception flottante de l'asymétrie des pouvoirs au cœur de la violence. L'analyse d'un corpus d'articles de la presse féminine permettra ensuite de souligner la façon dont la catégorie « pervers narcissique » tend à ériger

\footnotetext{
${ }^{1}$ CRESPPA-CSU, Université Paris 8 Saint-Denis, Université Paris Ouest Nanterre, pauline.delage@cnrs.fr

${ }^{2}$ Simone Korff-Sausse, « La femme du pervers narcissique », Revue française de psychanalyse, vol. 67, 2003, p. 926.

${ }^{3}$ Thierry Fiorilli, L'Express, 20 mars 2013, https://www.lexpress.fr/styles/psycho/pervers-narcissique-fred-et-marie-lespot-belge-qui-denonce-les-pervers-narcissiques 1233120.html

${ }^{4}$ Kathy Davis, "L'intersectionnalité, un mot à la mode. Ce qui fait le succès d'une théorie féministe », Les cahiers du CEDREF, $\mathrm{n}^{\circ}$ 20, 2015, URL : https://journals.openedition.org/cedref/827.

${ }^{5}$ Elisa Herman, Lutter contre les violences conjugales. Féminisme, travail social, politique publique, Rennes, Presses universitaires de Rennes, 2016 ; Pauline Delage, Violences conjugales. Du combat féministe à la cause publique, Paris, Presses de Sciences Po, 2017.
} 
les bornes (in-)égalitaires de la norme conjugale hétérosexuelle. Enfin, un détour états-unien donnera une idée de la manière dont l'influence du genre sur le narcissisme peut être abordée dans certains travaux de recherche en psychologie.

\section{Flottement dans la compréhension du genre}

En présentant les formes et les effets de la violence morale dans le cadre privé et au travail, le livre de Marie-France Hirigoyen, Le harcèlement moral. La violence perverse au quotidien, publié en 1998, a contribué à publiciser la catégorie de perversion narcissique en France ${ }^{6}$. La diffusion de cette catégorie a alors traduit et accéléré l'essor dans l'espace public du problème des violences psychologiques. À l'instar d'autres concepts psychologiques, notamment ceux centrés sur les effets psychiques de la victimation ${ }^{7}$, la notion de «perversion narcissique » désarrime la violence du corps pour l'appréhender comme une invasion et une atteinte psychiques. Elle participe alors non seulement de l'extension du domaine de la violence mais aussi de sa reformulation en des termes cliniques.

Dans son ouvrage, Marie-France Hirigoyen déploie une perspective de victimologie (à laquelle elle s'est formée au cours d'un séjour états-unien) : elle cherche à reconnaître la violence perverse et ses effets pour mieux soigner les victimes. Les liens entre les rapports de genre et la violence morale ne sont pas traités. Ainsi, agresseur et victime sont des termes sans cesse utilisés pour établir la place de chacun dans la violence, sans que l'un ou l'autre n'ait de genre ou que le rapport de pouvoir qui structure leur relation ne soit qualifié de la sorte. Ce sont l'espace dans lequel la violence a lieu, famille ou entreprise, et les liens qui unissent les victimes aux auteurs (filiaux, conjugaux, ou de subordination dans l'entreprise) qui organisent l'ouvrage pour comprendre la violence et développer des moyens pour en sortir.

Quelques éléments épars auraient pu donner lieu à une analyse plus systématique des rapports entre genre et violences. D'une part, les cas présentés dans la partie sur le couple sont exclusivement ceux où figurent un homme et une femme, cette dernière étant systématiquement la victime ${ }^{8}$. D'autre part, s'agissant de l'entreprise et du harcèlement entre collègues, on peut lire ceci : "Les groupes tendent à niveler les individus et supportent mal la différence (femme dans un groupe d'hommes, homme dans un groupe de femmes, homosexualité, différence raciale, religieuse ou sociale...). Dans certains corps de métier traditionnellement réservés aux hommes, il n'est pas facile pour une femme de se faire respecter quand elle arrive. Ce sont des plaisanteries grossières, des gestes obscènes, un mépris de tout ce qu'elle peut dire, le refus de prendre son travail en considération $»{ }^{9}$. C'est donc la différence, sexuée notamment, plutôt que l'asymétrie des pouvoirs qui est d'abord vue comme une source de harcèlement, même si le sexisme est bien reconnu dans la description qui est faite du type de violence morale. Quelques lignes plus loin, le déni des discriminations dans les organisations est en effet explicitement mentionné : "De nombreuses entreprises se révèlent incapables de faire respecter les droits minimaux d'un individu et laissent se développer, en leur sein, racisme et sexisme $\gg{ }^{10}$. Cependant le fait d'aborder la violence sous l'angle d'une de ses sous-catégories, à savoir la violence morale, plutôt que sous celui de la relation entre les protagonistes, se traduit dans certains passages par une mise en équivalence des comportements des hommes et des femmes. S'agissant de la famille, il est ainsi question de la violence des mères sur les enfants : se succèdent ainsi tour à tour des exemples d'inceste latent commis par une mère puis un père ${ }^{11}$.

\footnotetext{
${ }^{6} \mathrm{Si}$ le fondateur de cette notion est le psychiatre et psychanalyste Paul-Claude Racamier (1924-1996), d'autres travaux ont contribué à sa popularisation, notamment ceux d'Alberto Eiguer. Voir, en particulier, Alberto Eiguer, Le pervers narcissique et son complice, Paris, Dunod, 1989 (3 édition, 2003) ; Alberto Eiguer, Nouveaux portraits du pervers moral, Paris, Dunod, 2005 ; Alberto Eiguer, Les pervers narcissiques, Paris, Presses Universitaires de France, 2017. Sur cette circulation, voir, dans le présent dossier, la contribution de Marc Joly et de Corentin Roquebert.

${ }^{7}$ Stéphanie Pache, «L'histoire féministe de la "psychologisation des violences” », Cahiers du Genre, n 66, 2019, p. 5170.

${ }^{8}$ Marie-France Hirigoyen, Le harcèlement moral. La violence perverse au quotidien, Paris, Syros, 1998, p. 17-38

${ }^{9}$ Ibid., p. 60.

${ }^{10}$ Ibid., p. 61.

${ }^{11}$ Ibid., p. 51-52.
} 
La perspective clinique de l'auteure, qui se concentre sur un type de violence (psychologique) pour améliorer le traitement des victimes, tend à indifférencier les logiques sociales qui forgent les différentes configurations relationnelles (conjugale, familiale, salariale) dans lesquelles la violence peut se déployer. Plutôt que de traiter du couple hétérosexuel, où la domination masculine s'exprime en premier lieu, il est question d'une sous-catégorie de violence qui, paradoxalement, élargit l'ampleur et la force de frappe de la violence plutôt qu'elle ne la particularise. Ainsi l'asymétrie de genre est absente, ou passe au second plan. Cette focalisation a des effets semblables à ceux provoqués par l'usage de la catégorie " violence domestique » pour parler de violence conjugale, tels qu' on peut les observer dans le cadre de l'analyse de l'évolution de l'action publique en Suisse romande: l'élargissement des configurations relationnelles que traduit le passage de "violence conjugale » à « domestique » a renforcé l'invalidation de la grille d'analyse en termes de genre dans les politiques publiques $^{12}$.

Plus récemment, cette asymétrie est plus explicite lorsqu'il est question de violences dans le couple, comme dans le livre Femmes sous emprise, paru en 2005. En plaidant pour une meilleure appréhension de la violence psychologique dans le couple, ce sont bien les relations conjugales, hétérosexuelles, qui sont alors au cœur de l'ouvrage ${ }^{13}$. Les victimes de violence psychologique dans le couple sont alors explicitement identifiées comme des femmes, et la domination et le contrôle sont décrits comme les moteurs de cette violence. Dans le même ordre d'idées, l'introduction de l'article de 2009 "De la peur à la soumission» rappelle : "La violence au sein du couple a des effets dévastateurs pour les femmes qui en sont victimes, tant sur leur santé physique que sur leur santé mentale. Il s'agit d'un important problème de santé publique dont on ne perçoit trop souvent que l'aspect le plus visible du phénomène, à savoir la violence physique, alors que ce qui constitue la violence, c'est un mode de relation basé sur le contrôle et la domination ${ }^{14}$. En se concentrant sur les couples hétérosexuels, Marie-France Hirigoyen peut alors intégrer le sexisme à sa compréhension de la perversion narcissique. Mais ce rapport de pouvoir n'est pas systématiquement inséré dans son analyse générale des relations. Ainsi ce flottement conceptuel peut-il tout à fait donner lieu à des usages qui occultent le genre et renforcent des représentations masculinistes reposant sur une symétrisation de la violence ${ }^{15}$.

\section{Une figure repoussoir de la conjugalité}

Dans la presse féminine, nombre d'articles traitent des pervers narcissiques dans le cadre des relations conjugales. Sans faire de recensement systématique de ce corpus, un échantillon issu des sites internet d'Elle, Marie-Claire et Cosmopolitan illustre certains des motifs autour de ce thème : en s'appuyant souvent sur des discours experts, les travaux de Marie-France Hirigoyen notamment, la plupart des

\footnotetext{
${ }^{12}$ Pauline Delage, Marylène Lieber et Marta Roca i Escoda, Contrer les violences conjugales. Emergence et reconfigurations d'un problème public, Lausanne, Antipodes, 2020.

${ }^{13}$ Marie-France Hirigoyen, Femmes sous emprise. Les ressorts de la violence dans le couple, Paris, Oh éditions, 2005.

${ }^{14}$ Marie-France Hirigoyen, « De la peur à la soumission », Empan, 2009, nº 73, p. 24 à 30.

${ }^{15}$ Pour ne donner qu'un exemple de ce potentiel glissement, l'émission « Le monde en face » a réuni plusieurs expertes, dont Marie-France Hirigoyen, et un homme victime autour d'un débat, intitulé «Pervers narcissiques : Une violence invisible ", suite à un documentaire présentant les témoignages de victimes femmes. Pour lancer la discussion, l'animatrice Marina Carrère d'Encausse demande : «c'est important de rappeler que la violence morale, ce n'est pas l'apanage des hommes... ». L'une des invitées, Yvonne Poncet-Bonissol, psycho-clinicienne, renchérit alors : «ça se conjugue au masculin, comme au féminin, et je suis ravie que Charles (la victime présente sur le plateau) soit à nos côtés parce qu'on oublie souvent. On pense que ce sont surtout les femmes qui sont victimes de violence morale. Eh bien non (...). Les hommes sont vraiment pas préparés à ce genre de rencontres. Ils sont comme envoûtes, tout comme les femmes, et ils ne peuvent pas s'en sortir aussi aisément. » Et l'animatrice de conclure cet échange : "Et on imagine que c'est encore plus difficile pour eux d'en parler ». Invité à témoigner, Charles insiste sur le manque de structures destinés aux hommes et présente sa démarche : "Si je peux aider d'autres hommes à pouvoir parler de ça, pourquoi pas ?» Sans qu'il soit contredit pendant l'émission, l'un des piliers de la rhétorique masculiniste, qui consiste à souligner l'invisibilité des hommes victimes dans la problématisation de la violence dans le couple, voire les difficultés accrues qui se présenteraient à eux, est ici asséné. https://www.youtube.com/watch?v=Y1NPx sJ78g, consultée le 12 juillet 2020.
} 
articles doivent permettre aux lectrices de connaître le phénomène et d'identifier les pervers narcissiques. Ce faisant, ils leur prodiguent une grille d'analyse du couple.

Le récit de ces histoires conjugales, réelles ou imaginées, est souvent similaire. À l'occasion de la diffusion d'un documentaire sur ce thème, le psychanalyste Jean-Charles Bouchoux, auteur de Les pervers narcissiques, paru en 2008, est interrogé par Elle ${ }^{16}$ pour expliquer ce qui caractérise le comportement des pervers narcissique dans le cadre du couple et les étapes de l'emprise : "Tout commence toujours par de la séduction [...]. Une fois que vous avez confiance en lui, c'est à ce moment-là que cela commence, généralement très doucement, par une petite critique [...]. Et, petit à petit, vous rentrez dans un système, c'est comme une araignée qui tisse une toile autour de vous. » À la question «que faire pour sortir de son emprise? », il répond sans détour : " On le quitte, on s'en va et on coupe les ponts ». En combinant témoignages d'ex-compagnes de pervers narcissiques et un entretien avec Marie-France Hirigoyen, l'article de Marie-Claire "Amoureuse d'un pervers narcissique : l'enfer au quotidien » reprend la thématique de la rencontre rêvée devenue cauchemar à cause des humiliations quotidiennes qui dégradent l'estime qu'a la victime d'elle-même et sa capacité à faire des choix. En proposant un récit plus incarné, les témoignages de personnes célèbres ${ }^{17}$ ou anonymes ${ }^{18}$ renforcent la description de ce processus. Ils reviennent, d'abord, sur l'idée d'une rencontre merveilleuse, avant de décrire les moqueries répétées, les dénigrements, parfois les coups, ainsi que la peur. Il est aussi question du «déclic ${ }^{19}$, le moment où ces femmes réalisent que le comportement de leur compagnon est inadmissible, et où elles se tournent alors vers des institutions ou des personnes extérieures pour mettre fin à cette situation.

Autre déclinaison du thème de l'identification des pervers narcissiques, certains articles répertorient une liste de signes pour les reconnaître. De manière assez anecdotique, et évidemment absurde, un article de Marie-Claire rapporte une enquête publiée dans le Journal of Personality qui conclurait que l'épaisseur des sourcils d'un individu permettrait l'identification des pervers narcissiques ${ }^{20}$. Plus couramment fondé sur l'observation de comportements répétés, cet exercice diagnostic permet également de borner les limites des pratiques conjugales acceptables et inacceptables. Ainsi un article d'Elle introduit sa liste de 30 signes en précisant « si vous trouvez de 10 à 15 signes dans la liste et explique qu'entre 10 ou 15 points de la liste de comparaison [...], vous avez affaire à un simple manipulateur. Au-delà de 15 , vous êtes en proie à un pervers narcissique $»^{21}$.

La catégorie de pervers narcissique est le plus souvent utilisée sans distinction de genre, mais son usage suggère une description genrée silencieuse. Dans «Amoureuse d'un pervers narcissique », la réponse que fait Marie-France Hirigoyen à la question "peut-on tous être victimes? » le reflète particulièrement bien. Elle répond d'abord que cela concerne tout le monde en soulignant que «ce n'est pas tant la prédisposition psychologique de la victime que l'habileté du pervers qui compte »,

\footnotetext{
${ }^{16}$ Émilie Poyard, «Pervers narcissique : qui est-il vraiment ?», https://www.elle.fr/Societe/Interviews/Pervers$\underline{\text { narcissique-3009115, }}$, consulté en juillet 2020. Notons que le même auteur est interrogé par Marie-Claire: Claire Schneider, "Comment aider les victimes de pervers narcissiques », https://www.marieclaire.fr/,comment-aider-lesvictimes-de-pervers-narcissiques, 726182.asp, consulté en juillet 2020.

${ }^{17}$ Voir Géraldine Verheyen, « Lio : comment la chanteuse est sortie vivante de son couple avec un pervers narcissique et père de ses enfants ", Elle, https://www.elle.fr/People/La-vie-des-people/News/Lio-comment-la-chanteuse-est-sortievivante-de-son-couple-avec-un-pervers-narcissique-et-pere-de-ses-enfants-3726297, consulté en juillet 2020 ; Elisa Casson, « « Tu es grosse, tu as du gras sur le ventre » : Marion Bartoli se livre sur sa relation avec un pervers narcissique », Elle, https://www.elle.fr/Societe/News/Tu-es-grosse-tu-as-du-gras-sur-le-ventre-Marion-Bartoli-se-livre-sur-sa-relationavec-un-pervers-narcissique-qui-l-a-poussee-a-l-anorexie-3787359, consulté en juillet 2020.

${ }^{18}$ Sophie Le Bris, « Témoignage: «J'ai aimé un pervers manipulateur », Elle, https://www.elle.fr/Love-Sexe/Mon-mecet-moi/Articles/Temoignage-J-ai-aime-un-pervers-manipulateur-2000536, consulté en juillet 2020 ; Juliette Roche, « En couple avec un pervers narcissique... elles témoignent », Cosmopolitan, https://www.cosmopolitan.fr/victime-d-unpervers-narcissique,2004507.asp, consulté en juillet 2020.

${ }^{19}$ Ibid.

${ }^{20}$ Emmanuelle Ringot, «On pourrait reconnaître les pervers narcissiques grâce à un détail physique », https://www.marieclaire.fr/on-pourrait-reconnaitre-les-pervers-narcissiques-grace-a-un-detail-physique, 1268661.asp, consulté en septembre 2020. Le titre original de l'article: Miranda Giacomin et Nicolas O. Rule, «Eyebrows cue grandiose narcissism », Journal of Personality, vol. 87, n², 2019, p. 373-385.

21 Jérémy Patinier, «Pervers narcissique: quels signes permettent de les repérer?», https://www.elle.fr/Love$\underline{\text { Sexe/News/Demasquer-un-pervers-narcissique-30-attitudes-qui-ne-trompent-pas-3559235 }}$
} 
pour donner ensuite un exemple reposant sur la différenciation entre les hommes et les femmes : « Nombre de femmes veulent être réparatrices à tout prix pour leur homme, ce qui fait qu'elles restent, même malheureuses ${ }^{22}$. Comme dans Le harcèlement moral, le problème est pensé comme universel, mais les exemples sont particularisés.

L'indistinction implicite prévaut, mais, comme les textes s'adressent essentiellement à des femmes qui sont supposées hétérosexuelles, ils s'inscrivent dans la configuration conjugale hétérosexuelle où le partenaire masculin est pervers narcissique. Il s'agit de protéger les lectrices d'une relation amoureuse nuisible et destructrice. En outre, ces types de relation contreviennent à un idéal conjugal fondé sur un ethos égalitaire, "qui diffuse le principe de l'égalité entre les sexes en même temps que leur indépassable complémentarité ${ }^{23}$. Implicitement, c'est une autonomie encadrée par une bonne conjugalité que promeuvent ces articles. Outre le manque de reconnaissance et les marques de mépris, le contrôle sur l'activité, notamment salariée, et sur les relations amicales ou familiales de la victime sont autant de manifestations de la perversion narcissique. La perversion narcissique n'est pourtant pas envisagée directement comme un problème associé aux violences sexistes, à leur forte prévalence, à la persistance des inégalités et des différences de socialisation, ni même à l'enjeu des droits des femmes. Plutôt, la perversion narcissique et les pervers narcissiques apparaissent comme des cas limites de la norme conjugale, dont le respect mutuel est une valeur cardinale permettant d'articuler la différenciation des rôles avec l'idée d'égalité. Par le truchement de la dénonciation de la perversion narcissique, les excès de la conjugalité hétéronormée sont pointés et rendent cette norme conjugale effective : la division genrée du travail demeure un impensé, mais la dénégation et le mépris sont jugés problématiques. De la même manière, en étant associée à des termes renvoyant à ce qui est malsain et pathologique, comme ceux de toxicité ou de prédation, la perversion narcissique est érigée en repoussoir. Ainsi, il s'agit pour les femmes d'éviter ces figures exceptionnelles. Telle qu'elle est présentée dans les magazines, la quête d'autonomie des femmes est non seulement limitée et encadrée par la conjugalité hétérosexuelle, mais elle est également individualisée. Avec les avertissements, les conseils et les listes de signes que fournissent les articles, la responsabilité de trouver un bon conjoint et donc de former une bonne union incombe toujours aux femmes.

\section{Un narcissisme genré ?}

La « perversion narcissique », comme objet de discours médiatique et scientifique, semble entretenir avec la perspective en termes de genre une relation marquée par l'indétermination. Parallèlement, l'idée que le narcissisme puisse être genré transparaît explicitement de certains articles de presse et de psychologie. En 2015, plusieurs articles paraissent et insistent sur l'idée que le narcissisme serait masculin. Elle publie sur son site un article intitulé «Les femmes sont-elles moins narcissiques que les hommes? " ${ }^{24}$; Europe 1 fait de même avec le texte "C'est prouvé, les hommes sont plus narcissiques $»^{25}$, tout comme Top Santé, «Insolite, les hommes seraient plus narcissiques $»^{26}$. Ces articles font écho à l'article «Gender Differences in Narcissism : A Meta-Analytic review », publié

\footnotetext{
${ }^{22}$ Véronique Houguet, «Amoureuse d'un pervers narcissique: l'enfer au quotidien », Marie Claire, https://www.marieclaire.fr/,amoureuse-d-un-pervers-narcissique-une-relation-infernale,20296,692341.asp

${ }^{23}$ Isabelle Clair, «La découverte de l'ennui conjugal. Les manifestations contrariées de l'idéal conjugal et de l'ethos égalitaire dans la vie quotidienne de jeunes de milieux populaires », Sociétés contemporaines, 2011, vol. 83, p. 59-81.

24 Laura Boudoux, «Les femmes sont-elles moins narcissiques que les hommes?», Elle, https://www.elle.fr/Societe/News/Les-femmes-sont-elles-moins-narcissiques-que-les-hommes-2920728

25 Fabienne Cosny, «C'est prouvé. Les hommes sont plus narcissiques», Europe 1, mars 2015, https://www.europe1.fr/societe/C-est-prouve-les-hommes-sont-plus-narcissiques-779092

${ }^{26}$ Hélène Bour, «Insolite: les hommes seraient plus narcissiques que les femmes », Top Santé, mars 2015, https://www.topsante.com/forme-bien-etre/bien-dans-ma-peau/ma-personnalite/insolite-les-hommes-seraient-plusnarcissiques-que-les-femmes-245907
} 
dans le Psychological Bulletin la même année, qui interroge l'ampleur, les formes et la variabilité historique de la différence de genre dans le narcissisme ${ }^{27}$.

Si la notion de perversion narcissique est utilisée en France pour décrire des relations, notamment mais pas uniquement conjugales, génératrices de souffrance, celle de narcissisme circule davantage dans d'autres contextes, en particulier aux États-Unis ${ }^{28}$. Dans le Diagnostic and Statistical Manual of Mental Disorders (DSM), il est considéré comme un trouble de la personnalité depuis 1980 et a été redéfini en 2012. Le narcissisme se caractérise par un besoin d'être admiré, la folie des grandeurs, une haute estime de soi et l'absence d'empathie. Tout comme la notion elle-même, l'un des instruments d'évaluation du narcissisme, le Narcissistic Personnality Inventory (NPI), a fait l'objet de débats interrogeant sa capacité à mesurer les personnalités des hommes et des femmes. Ainsi, il a été démontré que certaines des caractéristiques du syndrome narcissique, comme le fait d'être sans scrupules (exploitative) ou le sentiment d'entitrement (entitlement), sont moins aisément affirmées par les femmes qui risquent de subir des sanctions négatives ${ }^{29}$.

Fondée sur une méta-analyse des recherches menées depuis 31 ans, « Gender Differences in Narcissism : A Meta-Analytic review » a testé trois principales hypothèses : 1. « en moyenne, les hommes présentent un niveau de narcissisme plus élevé que les femmes »; 2. "l'écart entre les hommes et les femmes diminue dans le temps »; 3. " en moyenne, les hommes présentent un niveau plus élevé de capacité à diriger (leadership) / autorité que les femmes ${ }^{30}$. D’autres études avaient montré que les hommes ont un niveau de narcissisme plus élevé et, selon l'American Psychological Association, près des trois quart des personnes présentant un trouble narcissique (narcissistic personnality disorder) sont des hommes ${ }^{31}$. Là, non seulement l'analyse rappelle que les hommes sont plus narcissiques que les femmes, mais aussi que les hommes sont plus susceptibles de «penser qu'ils sont eux-mêmes exceptionnels [special], ce qui doit leur conférer certains privilèges", et qu'ils ont "plus d'assurance, plus d'envie de diriger et de désir de pouvoir et d'autorité sur les autres »" Cependant, l'analyse ne souligne pas de réduction à travers le temps de l'écart entre les hommes et les femmes. Selon les auteur·e's, cela peut s'expliquer par le fait que les inégalités au travail n'ont pas suffisamment évolué depuis 1980, année marquant l'entrée du narcissisme dans le DSM et le début des enquêtes le mesurant.

L'intérêt de cette recherche, de notre point de vue, tient effectivement à l'affirmation d'un fondement théorique qui cherche à articuler ces dispositions psychologiques à la division du travail et à la socialisation de genre. La perspective adoptée dans l'article se veut bio-sociale : en se distinguant de l'essentialisme d'une part, du constructivisme social d'autre part, elle considère que les différences de la personnalité constatées entre les hommes et les femmes résultent des croyances

\footnotetext{
${ }^{27}$ Emily Grijalva, Daniel A Newman, Louis Tay, M. Brent Donnellan, Peter D. Harms, Richard W. Robins et Taiyi Yan, « Gender Differences in Narcissism: A Meta-Analytic Review », Psychological Bulletin, 2015, vol. 141, n², p. $261-310$.

${ }^{28}$ Ces variations sémantiques et conceptuelles reflètent des configurations disciplinaires différentes entre la France et les États-Unis, mais elles soulignent également une différenciation des problèmes. En effet, le narcissisme ne renvoie pas systématiquement aux violences psychologiques et peut être associé à des postures psychologiques jugées positivement, telles que l'estime de soi ou la capacité à diriger (leadership). Cependant, ce détour par les États-Unis ne cherche pas à étudier ces différences d'usage et de signification mais à rappeler que la lecture d'un trouble peut être arrimée à celle des rapports sociaux de pouvoir entre les sexes. Constantine Sedikides, Eric A. Rudich, Aiden P. Gregg, Madoka Kumashiro et Caryl Rusbult, «Are normal narcissists psychologically healthy?: Self-esteem matters », Journal of Personality and Social Psychology, 2004, vol. 87, n³, p. 400-416.

${ }^{29}$ Brian T. Tschanz, Carolyn C. Morf et Charles W. Turner, « Gender Differences in the Structure of Narcissism: A MultiSample Analysis of the Narcissistic Personality Inventory », Sex Roles, vol. 38, n 9-10, 1998, p. 863-870 ; Emily Grijalva, Daniel A Newman, Louis Tay, M. Brent Donnellan, Peter D. Harms, Richard W. Robins et Taiyi Yan, «Gender Differences in Narcissism: A Meta-Analytic Review », art. cit.

${ }^{30}$ Emily Grijalva, Daniel A Newman, Louis Tay, M. Brent Donnellan, Peter D. Harms, Richard W. Robins et Taiyi Yan, « Gender Differences in Narcissism: A Meta-Analytic Review », art. cit., p. 269-270.

${ }^{31}$ Jean M. Twenge, Sara Konrath, Joshua D. Foster, W. Keith Campbell, et Brad J. Bushman, B. « Further evidence of an increase in narcissism among college students », Journal of Personality, 2008, vol. 76, n4, p. 919-928; American Psychiatric Association., Diagnostic and statistical manual of mental disorders (4th ed., Text Revision), Washington, DC., 2000.

32 Emily Grijalva, Daniel A Newman, Louis Tay, M. Brent Donnellan, Peter D. Harms, Richard W. Robins et Taiyi Yan, « Gender Differences in Narcissism: A Meta-Analytic Review », p. 280.
} 
sur les rôles de genre qui s'ancrent dans la spécialisation biologique des sexes et la division genrée du travail et la socialisation ${ }^{33}$. Le fait que les femmes sont moins à même de se montrer meneuse tend à refléter et à alimenter la ségrégation horizontale du marché du travail. En creux, l'article insinue que les femmes manquent de certaines qualités narcissiques, telles que l'entitrement, pour atteindre des positions de pouvoir. Outre les implications cliniques de cette recherche, sa visée normative repose sur une perspective féministe que l'on peut qualifier de libérale : il s'agit de comprendre les conditions individuelles d'accès à des postes à responsabilité.

S'attarder sur cet article permet de rappeler que l'étude du narcissisme, même si c'est rare, peut être mise en lien avec celle du régime de genre, entendu comme les «agencements $\|^{34}$ des rapports sociaux de sexe. Le développement de savoirs psychologiques ne s'oppose pas nécessairement à la compréhension du contexte social et des effets des rapports de domination sur les comportements des individus et leurs trajectoires psychiques. Comme l'a rappelé Stéphanie Pache au sujet de la psychologie féministe : «La critique féministe consiste dans une large mesure à dénoncer l'absence de prise en considération de la société dans la production des théories et des connaissances psychologiques, ainsi que le caractère socialement construit - notamment par une construction scientifique - des différences sexuées ${ }^{35}$. À rebours de l'androcentrisme et du sexisme de leur discipline, des psychologues féministes ont en effet œuvré à la production de savoirs, notamment pour contrer ceux qui tendaient à responsabiliser et culpabiliser les femmes victimes de la violence qu'elles subissaient dans leur relation. En étudiant le traitement réservé à la variable genre dans les travaux sur le narcissisme et en les mettant en relation avec les enquêtes sur l'emploi aux États-Unis, l'article "Gender Differences in Narcissism » montre comment des dispositions psychologiques construisent le plafond de verre et les inégalités salariales.

Sans avoir d'écho médiatique en France, d'autres travaux ont interrogé les liens entre le narcissisme et les violences de genre. Le narcissisme a par exemple été vu comme un trait de personnalité favorisant la violence et la violence sexuelle ${ }^{36}$. Les effets de certaines composantes ou formes de narcissisme, plutôt que sa structure globale, ont également fait l'objet d'une analyse plus approfondie. Kathryn M. Ryan, Kim Weikel et Gene Sprechini ont ainsi travaillé sur l'influence du facteur être sans scrupules (exploitativeness) / entitrement (entitlement), du narcissisme grandiose et du narcissisme sexuel sur la violence au cours des relations amoureuses (courtship aggression) ${ }^{37}$. Si, selon l'étude, la violence est liée à ces deux formes de narcissisme, le genre joue un rôle important dans les jeux de corrélation entre certaines formes de narcissisme et la violence. Pourtant ce texte réduit la conception du genre à la répartition hommes / femmes, sans interroger plus avant les mécanismes sociaux qui le fonde et la structure. C'est le cas de l'article «Male Narcissism and Attitudes Toward Heterosexual Women and Men, Lesbian Women, and Gay Men: Hostility toward Heterosexual Women Most of All », paru dans Sex Roles en 2010, qui se penche sur la relation entre le narcissisme et le sexisme, en mobilisant différents indicateurs développés en psychologie sociale. Il montre que le narcissisme des hommes hétérosexuels est essentiellement associé à l'hostilité envers les femmes hétérosexuelles. À travers l'analyse du rapport aux femmes hétérosexuelles, mais aussi aux hommes gays et aux lesbiennes, c'est la structure de la domination masculine qui est étudiée pour comprendre le narcissisme.

\footnotetext{
33 Ibid., p. 282.

${ }^{34}$ Lorena Parini, Le système de genre. Introduction aux concepts et théories, Zurich, Seismo, 2006, p. 35 ; Raewyn W. Connell, Gender, Cambridge, Polity, 2002.

${ }^{35}$ Stéphanie Pache, « Entre science et politique : la question épistémologique dans l'histoire de la psychologie féministe », Recherches féministes, vol. 29, n¹, 2016, p. 43.

${ }^{36}$ Emily R. Mouilso et Karen S. Calhoun, «Personality and perpetration: Narcissism among college sexual assault perpetrators », Violence Against Women, vol. 22, n¹0, 2016, p. 1228-1242 ; Kyler Rasmussen, « Entitled vengeance: A meta-analysis relating narcissism to provoked aggression », Aggressive Behavior, 2016, vol. 42, n ${ }^{\circ}$, p. 362-379 ; Sinead Lambe, Catherine Hamilton-Giachritsis, Emily Garner et Julian Walker, «The Role of Narcissism in Aggression and Violence: A Systematic Review », Trauma, Violence and Abuse, vol. 19, n², 2018, p. 209-230.

${ }^{37}$ Kathryn M. Ryan, Kim Weikel et Gene Sprechini, « Gender Differences in Narcissism and Courtship Violence in Dating Couples », Sex Roles, vol. 58, 2008, n 11-12, p. 802-813.
} 
Ainsi, certains travaux en psychologie décortiquent une catégorie psychologique pour comprendre comment un régime de genre est institué, évolue et se pérennise.

\section{Et les hommes?}

Si la catégorie de perversion narcissique est souvent associée aux violences dans le couple et contribue à les rendre visibles, elle s'en distingue en se concentrant sur le contrôle et la souffrance psychiques d'une part, en renforçant un flou déjà diffus dans le rapport entretenu avec une analyse en termes de genre d'autre part. En creux, c'est bien une définition normative du couple hétérosexuel qui transparaît: la complémentarité et la hiérarchisation des rôles et des genres ne sont pas problématisées, elles sont bornées par leurs excès. Dans les articles scientifiques ou médiatiques que nous avons étudiés, les rapports de genre sont relativement absents - s'ils ne sont pas déniés -, quand l'importance du couple et le choix d'un bon conjoint sont, eux, indirectement réaffirmés. La conception et l'usage de cette catégorie ne permettent pas que les masculinités soient présentées comme un objet de connaissance psychologique, de témoignage public ou d'interrogation médiatique. Seule une figure repoussoir, pathologique, apparait.

Le narcissisme des hommes peut toutefois être pointé du doigt. Cette catégorie, proche de celle de perversion narcissique mais dont les contours définitionnels et cliniques diffèrent, circule outre-Atlantique; certains travaux qui la prennent pour objet interrogent parfois explicitement la différence de genre au cœur de ce phénomène. Outre des approches théoriques variées, du point de vue psychologique et de l'analyse du sexisme, un autre flou rend sans doute possible la diffusion de ces études. Contrairement à la perversion narcissique, le narcissisme n'est pas considéré comme un problème en soi : il est même souhaitable que certaines de ses composantes soient investies par les femmes pour lutter contre les inégalités sur le marché du travail. En définitive, ce sont donc souvent aux femmes de changer - de comportement ou de conjoint. 